\title{
Evaluation of swallowing function by two screening tests in primary COPD
}

\section{To the Editors:}

Swallowing is an important function to separate the airway and the oesophagus. Dysfunction of this mechanism may lead to aspiration of pathogenic oropharyngeal contents. The disorder of swallowing function can be evaluated by the simple two-step swallowing provocation test (STS-SPT) and repetitive saliva swallowing test (RSST) [1, 2]. Both screening tests are safe, rapid and of low cost. Recently, it has been reported that the swallowing dysfunction may be a risk factor for an exacerbation of chronic obstructive pulmonary disease (COPD) [3]. Therefore, detection of the swallowing dysfunction at an earlier stage of COPD may be very important in management of COPD patients and to prevent advancing stages of the disease. Swallowing dysfunction in advancing stages of COPD has been reported [3, 4]. However, it is unclear whether swallowing functions are disordered in primary COPD. The present study was conducted to elucidate swallowing function before exacerbation of COPD, especially at a mild stage, as defined by the Global Initiative for Chronic Obstructive Lung Disease (GOLD) classification [5], using the STS-SPT and the RSST. The present correspondence is presented as a preliminary to a prospective study investigating the probability of exacerbation.

Sixty-four patients (50 males and 14 females) with a diagnosis COPD (forced expiratory volume in $1 \mathrm{~s}$ (FEV1)/forced vital capacity $<70 \%$ ), who had experienced no exacerbation, were recruited from the outpatient clinic of Toyohashi Municipal Hospital, Toyohashi, Japan. Patients with cerebrovascular accident neuromyopathy and previous head and neck surgery were excluded. Ethical approval was obtained from the hospital and informed consent was obtained from all study subjects and controls.

The COPD patients were divided into the following three groups. 1) $\mathrm{FEV} 1 \geqslant 80 \%$ pred: mild; 2$) 50 \% \leqslant \mathrm{FEV} 1<80 \%$ pred: moderate; and 3) $30 \% \leqslant$ FEV $1<50 \%$ predicted: severe. Fifteen healthy subjects (six male and nine female) were recruited as a control group. The age of all of subjects was $73.2 \pm 3.6 \mathrm{yrs}$ $(n=79)$, and was not significantly different among the groups. All subjects underwent the STS-SPT and the RSST. In the STS$\mathrm{SPT}$, the subject was estimated as having abnormal swallowing when the swallowing response was not triggered within $3 \mathrm{~s}$ after a bolus injection of $2.0 \mathrm{~mL}$ of distilled water at the suprapharynx through a nasal catheter. In the RSST, swallowing function of the subject was estimated as abnormal when the number of saliva swallowings was less than three events during $30 \mathrm{~s}$. The data were analysed by Fisher's exact test. When significant effects were found at a $1 \%$ level, Tukey's multiple-range test was used.

In moderate and severe stages of COPD, the ratio of abnormal to normal swallowing patients estimated by the STS-SPT was

\begin{tabular}{|c|c|c|c|}
\hline TABLE 1 & $\begin{array}{l}\text { The number } \\
\text { estimated by } \\
\text { provocation te } \\
\text { saliva swallowi } \\
\text { chronic obstru }\end{array}$ & $\begin{array}{l}\text { rmal swal } \\
\text { ple two-s } \\
\text {-SPT) an } \\
\text { (RSST) ir } \\
\text { ulmonary }\end{array}$ & $\begin{array}{l}\text { atients } \\
\text { lowing } \\
\text { oetitive } \\
\text { stages of } \\
\text { (COPD) }\end{array}$ \\
\hline COPD stage & Patients $n$ & STS-SPT & RSST \\
\hline Normal (control) & 15 & $0^{\#}$ & $0^{\#}$ \\
\hline Mild & 12 & $3^{\#, 9}$ & $6^{*}$ \\
\hline Moderate & 35 & $17^{\circ}$ & $21^{\circ}$ \\
\hline Severe & 17 & $11^{\circ}$ & $11^{\circ}$ \\
\hline
\end{tabular}

higher than in the control group. In contrast, surprisingly, the RSST evaluated the ratio of abnormal to normal swallowing patients in the mild stage of COPD to be higher than the control; this was also the case in moderate and severe groups (table 1). However, the ratio was not significantly different between COPD stages.

The present results may suggest that both the STS-SPT and the RSST can be used to evaluate swallowing dysfunction before an exacerbation of COPD occurs and, furthermore, the RSST may even be valid at the mild stage of COPD. The present study also suggests that the swallowing dysfunction in COPD may begin in the mild stage and, therefore, aspiration should be monitored from the mild stage in COPD patients. For costperformance purposes, medical treatment of COPD patients is necessary at an earlier stage. The present study shows that is is possible to identify subjects who are at risk of developing COPD by an easy, low-cost method in a primary stage of the disease. Furthermore, the present correspondence could be developed as a study investigating the probability of exacerbation in COPD.

\section{K. Ohta*, K. Murata\#, T. Takahashi ${ }^{\mp}$, S. Minatani*, S. Sako* and Y. Kanada ${ }^{+}$ \\ ${ }^{*}$ Gifu Junior College of Health Science, "Dept of Biological Diversity and Resources, Gifu University, Gifu, "Dept of Audiology and Logopedics, Japan College of Rehabilitation and Welfare Professionals, Nagoya, and ${ }^{+}$Faculty of Rehabilitation School of Health Sciences, Fujita Health University, Toyoake, Japan.}

Correspondence: K. Murata, Dept of Audiology and Logopedics, Japan College of Rehabilitation and Welfare, 
2-14 Wakamiyacho, Nakamura-ku, Nagoya, Aichi 453-0023,

Japan. E-mail: crimson@dk2.so-net.ne.jp

Statement of Interest: None declared.

\section{ACKNOWLEDGEMENTS}

The authors thank K. Oshima for data analyses.

\section{REFERENCES}

1 Teramoto S, Matsuse T, Fukuchi Y, et al. Simple two-step swallowing provocation test for elderly patients with aspiration pneumonia. Lancet 1999; 353: 1243.
2 Oguchi K, Saitoh E, Baba M, et al. The repetitive saliva swallowing test (RSST) as a screening test of functional dysphagia. (2) Validity of RSST. Jpn J Rehabil Med 2000; 37: 383-388.

3 Kobayashi S, Kubo H, Yanai M. Impairment of the swallowing reflex in exacerbations of COPD. Thorax 2007; 62: 1017.

4 Mokhlesi B, Logemann JA, Rademaker AW, et al. Oropharyngeal deglutition in stable COPD. Chest 2002; 121: 361-369.

5 www.goldcopd.com GOLD Global Strategy for the Diagnosis, Management, and Prevention of Chronic Obstructive Pulmonary Disease, 2008. Date last updated: January 26, 2009. Date last accessed: January 26, 2009.

\section{Histological findings of the computed tomography halo in pulmonary sarcoidosis}

In 1974, SAHN et al. [1] described a patient with sarcoidosis who presented with a fine acinar rosette pattern of infiltrate on chest radiograph and alveolar filling with mononuclear cells in the biopsied specimen. In 1978, SHIGEMATSU et al. [2] reported the presence of noncaseating epithelioid granulomas in the alveolar spaces in patients with radiographic findings similar to the previous report. They considered these lesions to represent the early stage of sarcoidosis. Although there seems to be no definite relationship between the stage of sarcoidosis and the anatomical extent of the granulomas, sarcoid granulomas in the alveoli are rarely found. Nowadays, in the era of high-resolution computed tomography (HRCT), ground-glass attenuations are sometimes found in sarcoidosis that could fit

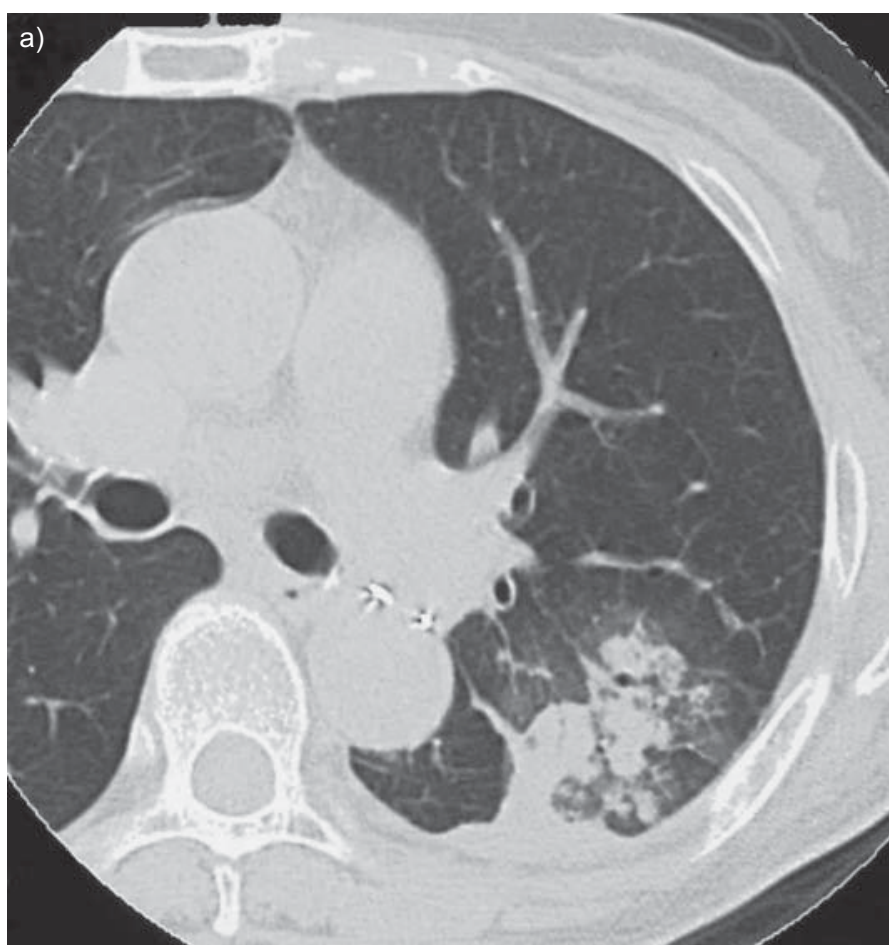

the "acinar pattern" proposed by SAHN et al. [1] and SHIGEMATSU et al. [2].

In 2004, MARTEN et al. [3] described the presence of groundglass attenuation around solid nodules, the halo sign, on computed tomography $(\mathrm{CT})$ of a patient with sarcoidosis.

Herein, we report a case of sarcoidosis presenting a CT halo sign that was surgically biopsied. The pathological/radiological correlation of sarcoidosis with the halo sign is discussed.

A 69-yr-old female noticed general fatigue. She had a past history of left lower lobectomy due to adenocarcinoma of the lung 8 yrs prior to admission. She was admitted to our hospital

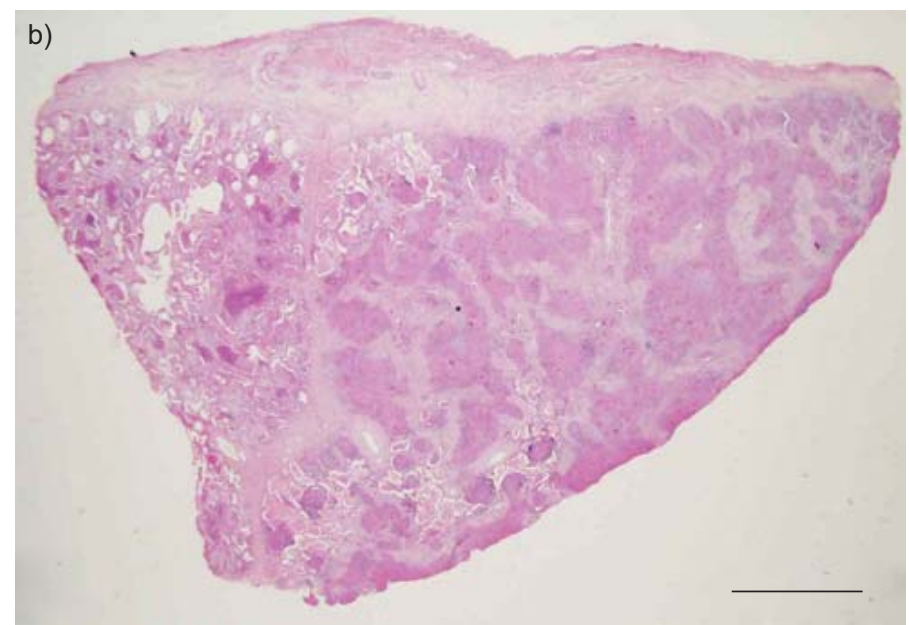

FIGURE 1. a) High-resolution computed tomography (CT) demonstrating multiple nodules and ground-glass opacities surrounding the nodules (i.e. halo sign). b) The biopsied sample on low-power microscopy (haematoxylin and eosin stain). The right two-thirds of the specimen represent the solid area corresponding to an area of the multiple nodules visible on the CT. The left third of the specimen represents the area of the halo sign visible on the CT. Scale bar $=2.0 \mathrm{~mm}$. 